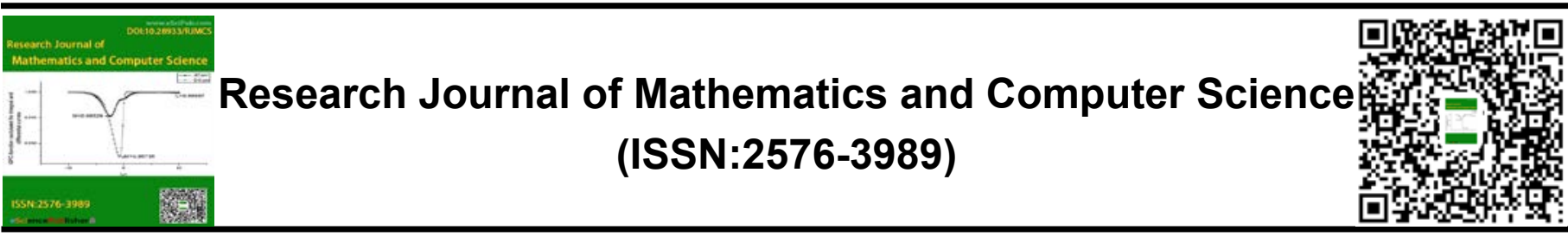

\title{
Chaotic Random Sequence Generated from Tent Map on Variant Maps
}

Yifeng Zheng ${ }^{1}$, Jeffrey Zheng ${ }^{1}$

${ }^{1}$ School of Software, Yunnan University, Kunmming 650091, China

\begin{abstract}
Chaotic sequences, have being widely used in mobile communications and cyberspace security. Using the sequences, various stochastic analysis schemes are developed. The Tent map is one of the widely used chaotic maps with good ergodic uniformity. In this paper, the variant maps are used to illustrate the Tent *Correspondence to Author: Jeffrey Zheng School of Software, Yunnan University, Kunmming 650091, Chichaotic sequence under different lengths and control parameters to show the statistical characteristics of sequences. Results are shown that when the control parameter of Tent map is close to 0.5 , the generated sequences have the symmetrical distribution, that is more stable if the sequence is longer.

na

Keywords: tent map, chaotic random sequence, variant map, visual testing.

How to cite this article:

Yifeng Zheng and Jeffrey Zhenga Chaotic Random Sequence Generated from Tent Map on Variant Maps. Research Journal of Mathematics and Computer Science, 2018; $2: 12$

\section{eSciencePublisher}

eSciPub LLC, Houston, TX USA. Website: http://escipub.com/
\end{abstract}




\section{Introduction}

Pseudo-random bit sequences have a wide range of applications, in the field of cryptography and communications[1]. Chaotic maps have the features of ergodicity, sensitivity to initial conditions and sensitivity to control parameters[2], can be implemented in higher speed for various algorithms. Tent map is the simplest chaotic maps, one of the best-known discrete dynamical systems[3][4]. It can be used in optimization algorithms[5], key stream generation[6], chaotic block cryptosystem[7] and digital noise production[8].

$$
x_{n+1}=\left\{\begin{aligned}
\frac{x_{n}}{a} & 0 \leqslant x_{n}<a \\
\frac{1-x_{n}}{1-a} & a \leqslant x_{n} \leqslant 1
\end{aligned}\right.
$$

The basic symbols are as follows:

(1) $x_{0}$ : the initial value

(2)n: the number of iterations

(3) $x_{n}$ : the $\mathrm{n}$-th value

(4) $x_{n+1}$ : the $(n+1)$-th value

(5)a: the control parameter for fractal

\section{Variant Map}

Variant map is a visual technology using multiple statistical probability distributions as visual maps to handle multiple $0-1$ vectors in its phase space on variant framework[10]. Different applications are explored using variant maps on sequences
From a distribution viewpoint, the randomness of Tent chaotic sequences may not be ideal[9]. Such measures do not reflect the various statistical distributions of Tent maps under different parameters. In this paper, chaotic sequences generated by different parameters and iteration times are tested and observed on variant maps, to illustrate the statistical distribution of Tent map systematically.

\section{Tent Map}

Lemma 2.1. Tent map is an one-dimensional piecewise linear map. The mapping form of Tent map is as follows:

of DNA data[11], bat echolocation call[12] and stream ciphers[13].

Various variant maps are shown in Figure 1, four maps generated from one sample sequence as (a)-(d). In map (a), a 2DPQ map is shown in 3D distribution; in map (b), a $2 \mathrm{DPQ}$ map is shown as a $2 \mathrm{D}$ color map; in map (c), the 2DPQ map is projected in the horizontal direction as a 1DP map in a normal bell shape; in map (d), the $2 \mathrm{DPQ}$ map is projected in the horizontal direction as a 1DQ map in a narrow bell shape respectively.

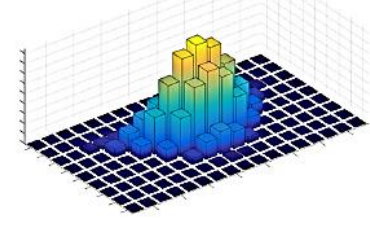

(a)

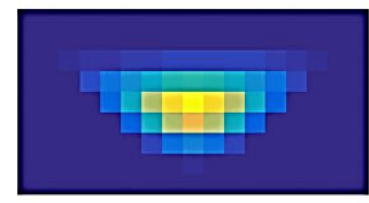

(b)

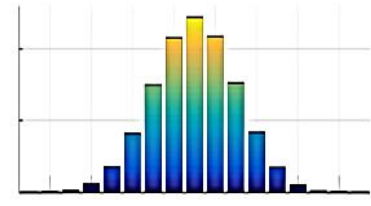

(c)

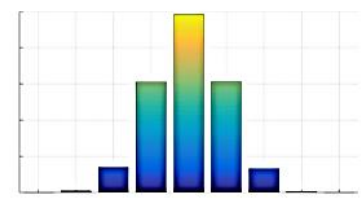

(d)

Figure 1: Variant Maps Outcomes (a)2DPQ-3D (b)2DPQ (c)1DP (d)1DQ

The basic symbols are used in this paper as follows:

(1)S: an input 0-1 sequence

(2) $s_{i}$ : the i-th element of $S$

(3) $S_{j}$ : the j-th segment of $S$

(4)N: the length of $S$

(5)m: the length of a segment
(6)M: the number of segments

(7)p: the number of $1 \mathrm{~s}$ elements in the segment (8)q: the number of 01s patterns in the segment

\section{Testing Procedure}

This testing procedure is shown in Figure 2 and composed of five steps:

Step 1: Initialization; 
Step 2: Generation;

Step 3: Segmentation;
Step 4: Measurements;

Step 5: Visual Maps.

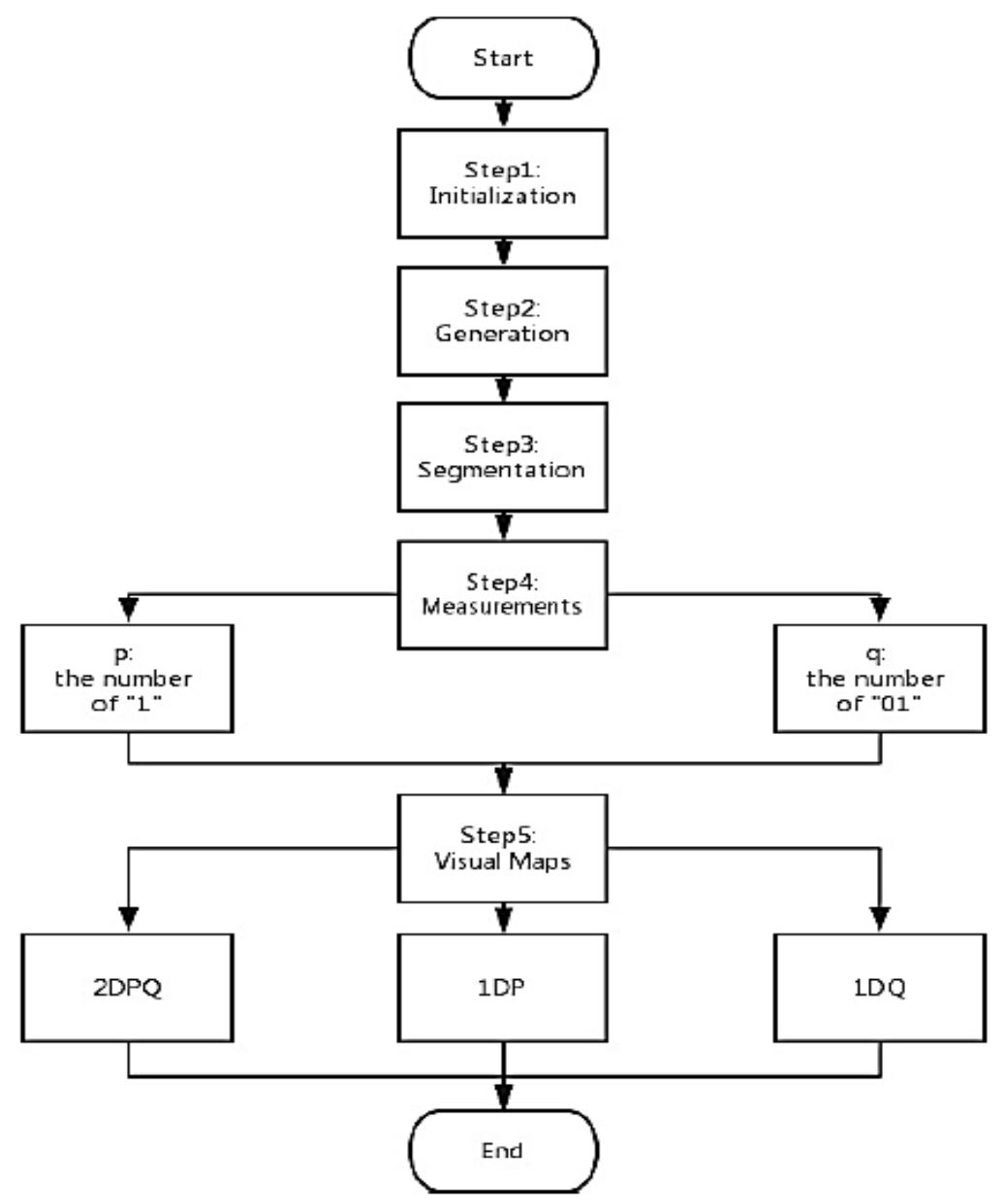

Figure 2: Testing Procedure

Each step can be described as follows:

\section{Step 1: Initialization}

Using the condition shown in Lemma 2.1, different initial parameters are selected: the initial value $x_{0}$, controls parameter a, iteration number $\mathrm{n}$, generating a chaotic sequence with length $\mathrm{N}$.

\section{Step 2: Generation}

Applying the initial parameters, a 0-1 random sequence $S$ is generated with length $N$.

$$
S=\left(s_{0}, s_{1}, \ldots, s_{i}, \ldots, s_{N-1}\right), s_{i} \in\{0,1\}, 0 \leqslant i<N
$$

\section{Step 3: Segmentation}

Make $m$ length segmentation on the $0-1$ flow of
$\mathrm{S}$, to divide it into $\mathrm{M}$ segments and each segment has $m$ elements.

$$
\begin{gathered}
M=\left[\frac{N}{m}\right] \\
S=\left(S_{0}, S_{1}, \ldots, S_{j}, \ldots S_{M-1}\right), 0 \leqslant j<M \\
S_{j}=\left(s_{j \times m}, s_{j \times m+1}, \ldots, s_{j \times m+k}, \ldots, s_{j \times m+m-1}\right), 0 \leqslant k<m, 0 \leqslant j \times m+k<N
\end{gathered}
$$




\section{Step 4: Measurements}

Two measurements $p_{j}$ and $q_{j}$ are calculated from each segment $S_{j}$ of $\mathrm{S}$, that is the number of 1 and 01. Pay attention to the 01 on tail head

$$
\begin{aligned}
& p_{\text {set }}=\left\{p_{0}, p_{1}, \ldots, p_{j}, \ldots, p_{M-1}\right\}=\left\{p_{j}\right\}_{j=0}^{M-1}, 0 \leqslant j<M \\
& q_{\text {set }}=\left\{q_{0}, q_{1}, \ldots, q_{j}, \ldots, q_{M-1}\right\}=\left\{q_{j}\right\}_{j=0}^{M-1}, 0 \leqslant j<M
\end{aligned}
$$

\section{Step 5: Visual Maps}

Order segments by its value of $p$ and $q$, types of diagrams can be created: Firstly, 1DP and $1 \mathrm{DQ}$

are one-dimension maps, which is sorted from $\left\{\mathrm{p}_{j}\right\}_{j=0}^{M-1}$ and $\left\{q_{j}\right\}_{j=0}^{M-1}$. S Secondly, twodimension $2 \mathrm{DPQ}$ is

sorted from a pair of measurements

$$
\begin{aligned}
& \left\{\left(p_{j}, q_{j}\right)\right\}_{j=0}^{M-1} \text {, created from }\left\{p_{j}\right\}_{j=0}^{M-1} \text { and } \\
& \left\{q_{j}\right\}_{j=0}^{M-1} .
\end{aligned}
$$

\section{Test Results}

In this paper, we use $2 \mathrm{DPQ}$ to show the distribution characteristic of Tent map. The test sample is a binary sequence cipher stream generated by Tent map. The initial value $x_{0}$ is fixed at 0.314 and the control parameter $a$ is from 0 to 1 , which satisfies the chaotic condition. The sample size ranges from $1 \mathrm{~KB}$ to $100 \mathrm{MB}$.

\subsection{Test Results on Different Segment Lengths}

The parameters and the results are shown in Table 1 and Figure 3 respectively:

Table 1: Relevant Parameters for Segment Lengths

\begin{tabular}{ccccc}
\hline Segment Length $m$ & Initial Value $x_{0}$ & Control Parameter $a$ & Length $N$ & Figure 3 \\
\hline 8 & & & & (a) \\
16 & & & & (b) \\
32 & & & & (c) \\
64 & 0.314 & 0.499 & $10,000,0000$ & (d) \\
128 & & & & (e) \\
256 & & & (f) \\
\hline
\end{tabular}

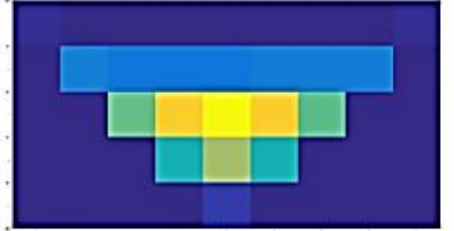

(a)

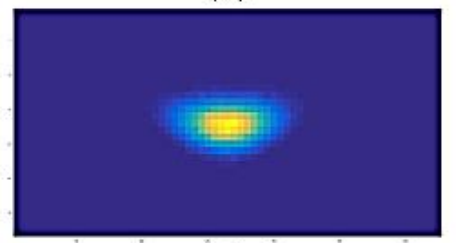

(d)

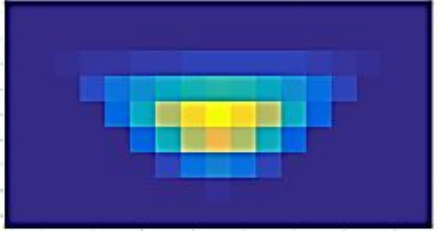

(b)

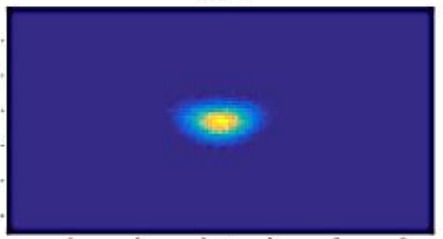

(e)

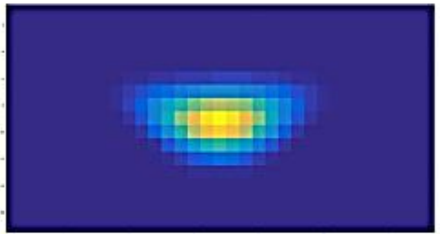

(c)

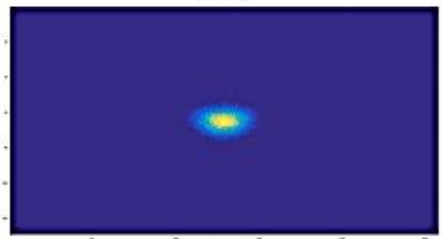

(f)

Figure 3: Test Results on (a)m=8 (b)m=16 (c)m=32 (d)m=64 (e)m=128 (f)m=256 
Yifeng Zheng and Jeffrey Zheng, RJMCS, 2018; 2:12

5.2. Test Results on Different Sequence Lengths
The parameters and the results are shown in Table 2 and Figure 4 respectively:

Table 2: Relevant Parameters for Sequence Lengths

\begin{tabular}{ccccc}
\hline Segment Length $m$ & Initial Value $x_{0}$ & Control Parameter $a$ & Length $N$ & Figure 4 \\
\hline & & 1,000 & (a) \\
16 & \multirow{2}{*}{0.314} & 0.499 & 10,000 & (b) \\
& & 100,000 & (c) \\
& & $1,000,000$ & (d) \\
& & $10,000,000$ & (e) \\
& & $100,000,000$ & (f) \\
\hline
\end{tabular}

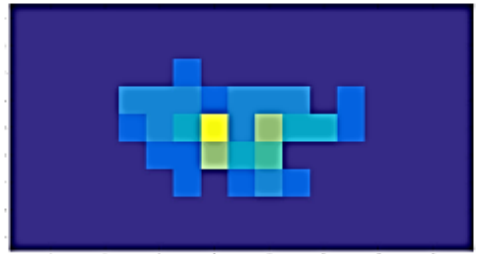

(a)

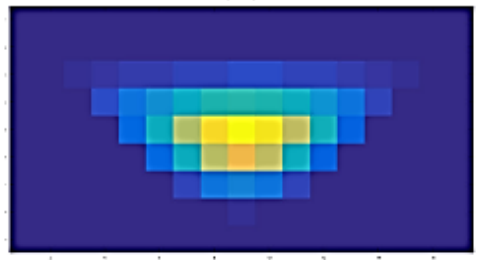

(d)

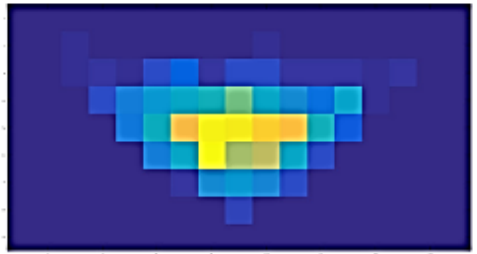

(b)

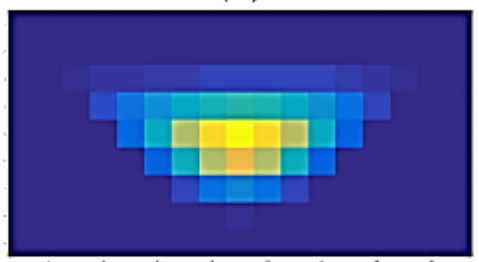

(e)

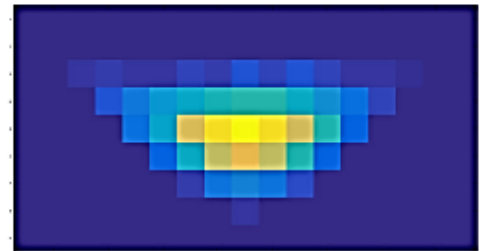

(c)

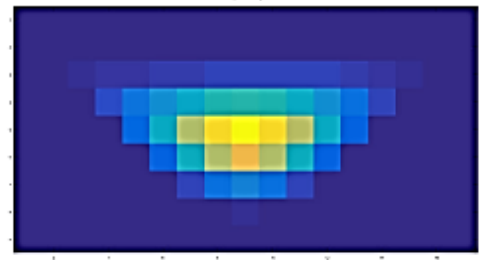

(f)

Figure 4: Test Results on (a) $\mathrm{N}=1,000$ (b) $\mathrm{N}=10,000$ (c) $\mathrm{N}=100,000$ (d) $\mathrm{N}=1,000,000$ (e) $\mathrm{N}=10,000,000$ (f) $\mathrm{N}=100,000,000$

\subsection{Test Results on Different Control} Parameters
The parameters and the results are shown in Table 3 and Figure 5 respectively:

Table 3: Relevant Parameters for Control Parameters

\begin{tabular}{ccccc}
\hline Segment Length $m$ & Initial Value $x_{0}$ & Control Parameter a & Length $N$ & Figure 5 \\
\hline & & 0.001 & (a) \\
& & 0.1 & (b) \\
& & 0.2 & (c) \\
& & 0.3 & (d) \\
& & 0.4 & (e) \\
& 0.314 & 0.499 & $10,000,000$ & (f) \\
& & 0.6 & & (g) \\
& & 0.7 & (h) \\
& 0.8 & (i) \\
& 0.9 & (j) \\
& & 0.999 & (k) \\
\hline
\end{tabular}

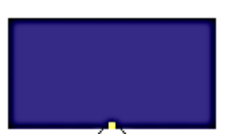

(a)

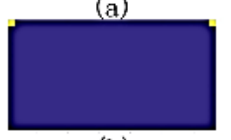

(k)

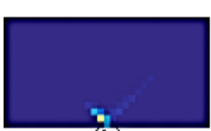

(b)

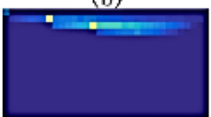

(j)

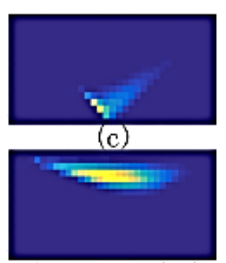

(i)

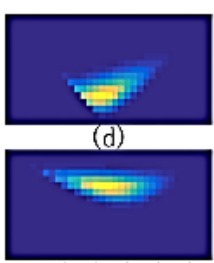

(h)

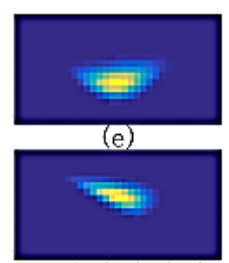

(g)

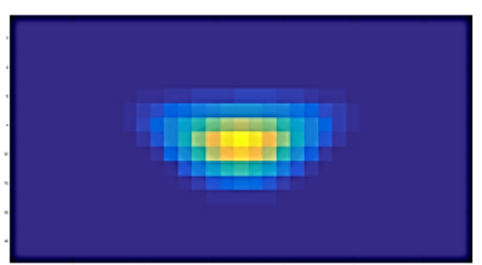

(f)

Figure 5: Test Results on (a)a=0.001 (b) $a=0.1$ (c) $a=0.2$ (d) $a=0.3(e) a=0.4(f) a=0.499$ (g) $a=0.6(h) a=0.7$ (i) $a=0.8$ (j) $a=0.9(k) a=0.999$ 


\section{Result Analysis}

When only $\mathrm{m}$ is changed, all 2DPQ maps are shown in left-right reflection symmetry and most of the segments are concentrated at the center, regardless of the value of $m$. For a larger $m$, more concentrated distributions are shown in the central region.

When input sequences are changed, a longer sequence is shown in a more stable distribution. It seems that when $\mathrm{N}$ reaches millions, variant maps Figure 4 (d)-(f) are shown in similar as one distribution.

When only $a$ is changed, three convergent points $\mathrm{a}=0, \mathrm{a}=0.5, \mathrm{a}=1$ are interesting, three closed parameter values $\mathrm{a}=0.001, \mathrm{a}=0.499, \mathrm{a}=0.999$ are chosen. It can be seen that when a approaches 0 , the distribution map converges to a single point shown in Figure 5(a).

When a changes from 0 to 0.5 , shown in Figure $5(a)-(f)$, the convergent point gradually diverges and the distribution tends to be stable, which shows that as the control parameter is close to

\section{References}

[1] XING-YUAN WANG, YI-XIN XIE, A DESIGN OF PSEUDO-RANDOM BIT GENERATOR BASED ON SINGLE CHAOTIC SYSTEM, J. International Journal of Modern Physics C, 2012, 23(03):1250024-.

[2] Lian S, Sun J, Wang Z, A block cipher based on a suitable use of the chaotic standard map, J. Chaos Solitons \& Fractals, 2005, 26(1):117-129.

[3] Habutsu T, Nishio Y, Sasase I, A Secret Key Cryptosystem By Iterating A Chaotic Map, J. Advances in Cryptology ł Eurocrypt, 1995, 547(1):127-140.

[4] Campos-Cantn I, Campos-Cantn E, Murguła J S, A simple electronic circuit realization of the tent map, J. Chaos Solitons \& Fractals, 2009, 42(1):12-16.

[5] Liang S, Hao Q, Li J, Chaotic optimization algorithm based on Tent map, J. Control \& Decision, 2005, 20(2).

[6] Beijing, A class of topologically conjugated chaotic maps of tent map to generate independently and uniformly distributed chaotic key stream, J. Acta Physica Sinica, 2013, 62(12):120501-464.

[7] Martłnez-Nonthe J A, Castaneda-Solłs A, DłazMndez A, Chaotic block cryptosystem using high precision approaches to tent map, J. Microelectronic Engineering, 2012, 90(2):168-172.
0.5 , the map is shown the best stability of normal distributions in Figure $5(\mathrm{f})$.

As a changes from 0.5 to 1 , relevant maps are changed gradually from left-right symmetric distributions

to be two separated concentrations to identified two points on both 0 and 1 positions shown in Figure 5(k).

\section{Conclusion}

In this paper, variant maps are applied to the chaotic random sequence based on Tent map, to show the statistical distributions of Tent map systematically. The distribution is stable when the control parameter is close to 0.5 if the sequence is long enough. The convergent points of the Tent map are identified and verified with the numeric condition, which can be shown from variant maps. From what has been discussed, the variant maps can be applied to Tent chaotic map. Since this is only an initial testing work, further systematic testing and evaluations are required to explore complex behaviors of Tent chaotic sequences.

[8] Palacios-Luengas L, Delgado-Gutirrez G, CruzIrisson M, Digital noise produced by a non discretized tent chaotic map, J. Microelectronic Engineering, 2013, 112(12):264-268.

[9] Khan M K, Zhang J, Investigation on Pseudorandom Properties of Chaotic Stream Ciphers, C. IEEE International Conference on Engineering of Intelligent Systems. IEEE, 2006, 1-5.

[10] Zheng J Z J, Zheng $\mathrm{C} \mathrm{H}$, A framework to express variant and invariant functional spaces for binary logic, J. Frontiers of Electrical \& Electronic Engineering in China, 2010, 5(2):163-172.

[11] Zheng J, Zhang W, Luo J, Variant Map System to Simulate Complex Properties of DNA Interactions Using Binary Sequences, J. Advances in Pure Mathematics, 2013, 3(7A):5-24.

[12] Heim D, Heim Olga, PA Zeng, Zheng Jeffrey, Successful Creation of Regular Patterns in Variant Maps from Bat Echolocation Calls, J. Biological Systems: Open Access, 2016, 5:2.

[13] Zheng J, Novel pseudo random number generation using variant logic framework, C. Proceedings of the 2nd International Cyber Resilience Conference, 2011, 100-104. 